\title{
Oscillation criteria for a class of third-order Emden-Fowler delay dynamic equations with sublinear neutral terms on time scales
}

Zhiyu Zhang $^{1 *}$ and Ruihua Feng ${ }^{2}$

"Correspondence:

18903460018@163.com

1 Department of Mathematics,

Taiyuan Institute of Technology,

Taiyuan, China

Full list of author information is

available at the end of the article

\section{黛 Springer}

\begin{abstract}
In this paper, we study the oscillation of a class of third-order Emden-Fowler delay dynamic equations with sublinear neutral terms on time scales. By using Riccati transformation and integral inequality, we establish several new theorems to ensure that each solution of the equation oscillates or asymptotically approaches zero, and the results in the literature are supplemented and extended. Examples are given to illustrate our main results.
\end{abstract}

Keywords: Time scale; Oscillation; Third-order; Sublinear neutral term; Emden-Fowler delay dynamic equation

\section{Introduction}

In this paper, we are concerned with the oscillation and asymptotic behavior of the thirdorder Emden-Fowler delay dynamic equation with sublinear neutral terms of the form

$$
\left(b(t)\left(\left(a(t)\left(x(t)+p(t) x^{\alpha}(\delta(t))\right)^{\Delta}\right)^{\Delta}\right)^{\gamma}\right)^{\Delta}+f(t, x(\tau(t)))=0, \quad t \in\left[t_{0}, \infty\right]_{\mathbb{T}}
$$

on a time scale $\mathbb{T}$.

Throughout this paper, the following assumptions are tacitly satisfied:

$\left(A_{1}\right) a(t), b(t), p(t) \in C_{r d}^{1}(\mathbb{T}, \mathbb{R})$, and $b^{\Delta}(t) \geq 0,0<p(t) \leq p<1$,

$$
\int_{t_{0}}^{\infty} \frac{1}{a(t)} \Delta t=\infty, \quad \int_{t_{0}}^{\infty} b^{-\frac{1}{\gamma}}(t) \Delta t=\infty
$$

$\left(A_{2}\right) \alpha, \beta, \gamma$ are quotients of odd positive integers, where $\beta \geq \gamma, 0<\alpha \leq 1$;

$\left(A_{3}\right) \delta(t), \tau(t) \in C_{r d}^{1}(\mathbb{T}, \mathbb{R})$ such that $\tau(t) \leq t, \delta(t) \leq t, \lim _{t \rightarrow+\infty} \tau(t)=\lim _{t \rightarrow+\infty} \delta(t)=\infty$.

$\left(A_{4}\right) f \in C(\mathbb{T} \times \mathbb{R}, \mathbb{R})$ is assumed that $u f(t, u)>0$ for $u \neq 0, t \in \mathbb{T}$, and there exists a function $q(t) \in C_{r d}^{1}(\mathbb{T}, \mathbb{R})$ such that $\frac{f(t, u)}{u^{\beta}} \geq q(t)$ for $u \neq 0, t \in \mathbb{T}$.

For the basic theory and notation of calculus on the time scale $\mathbb{T}$, we can see, for instance, the monograph [1], which systematically gives the definition of delta (or nabla) differentiation, the basic algorithm, and the important properties, such as the following:

(c) The Author(s) 2021. This article is licensed under a Creative Commons Attribution 4.0 International License, which permits use, sharing, adaptation, distribution and reproduction in any medium or format, as long as you give appropriate credit to the original author(s) and the source, provide a link to the Creative Commons licence, and indicate if changes were made. The images or other third party material in this article are included in the article's Creative Commons licence, unless indicated otherwise in a credit line to the material. If material is not included in the article's Creative Commons licence and your intended use is not permitted by statutory regulation or exceeds the permitted use, you will need to obtain permission directly from the copyright holder. To view a copy of this licence, visit http://creativecommons.org/licenses/by/4.0/. 
Assume that $f: \mathbb{T} \rightarrow \mathbb{R}$ is a function, and let $t \in \mathbb{T}^{k}$. We define $f^{\Delta}(t)$ to be the number, provided it exists, with the property that, for any $\epsilon>0$, there exists a neighborhood $U$ of $t, U=(t-\delta, t+\delta) \cap \mathbb{T}$ for some $\delta>0$ such that

$$
\left|f(\sigma(t))-f(s)-f^{\Delta}(t)(\sigma(t)-s)\right| \leq \epsilon|\sigma(t)-s| \quad \text { for all } s \in U
$$

We call $f^{\Delta}(t)$ the delta or Hilger derivative of $f$ at $t$. We say that $f$ is delta or Hilger differentiable, shortly differentiable, in $\mathbb{T}^{k}$ if $f^{\Delta}(t)$ exists for all $t \in \mathbb{T}^{k}$. The function $f^{\Delta}: \mathbb{T} \rightarrow \mathbb{R}$ is said to be the delta derivative or Hilger derivative, shortly derivative, of $f$ in $\mathbb{T}^{k}$, where $\sigma(t)=\inf \{s \in \mathbb{T}: s>t\}$ is called the forward jump operator. The properties of the delta $(\Delta)$ derivative and the knowledge of nabla $(\nabla)$ derivatives can be found in the monograph [1], which is omitted here.

Since we are interested in oscillation and asymptotic behavior of solutions to equation, we assume that the time scale $\mathbb{T}$ is unbounded. In this paper, we only consider those solutions of Eq. (1.1) which satisfy $\sup \left\{|x(t)|: t \in[\mathbb{T}, \infty)_{\mathbb{T}}\right\}>0$ and assume that such solutions exist. A solution of (1.1), which is nontrivial for all large $t$, is said to be oscillatory if it is neither eventually positive nor eventually negative. Otherwise, it is called nonoscillatory.

The theory of dynamic equations on time scales not only unifies the theory of differential equations and difference equations, but it also extends these classical cases to cases "in between", e.g., $\mathbb{T}=q^{\mathbb{N}_{0}}, \mathbb{T}=h \mathbb{Z}$, and other different time scales, which have wide application value in quantum theory, mathematical, theoretical, and chemical physics and ecology. Therefore, the study of the properties of dynamic equations on time scales has become a hot topic. In the past 20 years, there have been a lot of research results on the oscillation of dynamic equations. As a matter of fact, Eq. (1.1) is a natural generalization of the half-linear/Emden-Fowler dynamic equation (including related differential equation) which arises in a variety of real world problems such as in the study of $p$-Laplace equations, non-Newtonian fluid theory, the turbulent flow of a polytrophic gas in a porous medium, and so on; see, e.g., the papers [2-5] for more details. In this paper, our main purpose is to study the oscillation and asymptotic behavior of Eq. (1.1). In order to enlighten the research ideas, we briefly review the following research progress and important results of this problem.

Agarwal et al. [6] investigated the oscillation of a certain class of second-order differential equations with a sublinear neutral term

$$
\left(a(t)\left[x(t)+p(t) x^{\alpha}(\tau(t))\right]^{\prime}\right)^{\prime}+q(t) x(\sigma(t))=0, \quad t>t_{0}>0,
$$

where $0<\alpha<1$ is a ratio of odd positive integers.

Dzurina et al. [3], Grace and Graef [7], and Tamilvanan et al. [8] established sufficient conditions for the oscillation of all solutions of a nonlinear differential equation

$$
\left(a(t)\left(x(t)+p(t) x^{\alpha}(\tau(t))\right)^{\prime}\right)^{\prime}+q(t) x^{\beta}(\sigma(t))=0, \quad t \geq t_{0},
$$

where $\alpha$ and $\beta$ are ratios of odd positive integers.

Agarwal et al. [9] and Erbe et al. [10] established several Hille and Nehari type criteria for the third-order dynamic equation

$$
x^{\Delta \Delta \Delta}(t)+p(t) x(\tau(t))=0, \quad t \in \mathbb{T}, t \geq t_{0} .
$$


Agarwal et al. [11, 12], Han et al. [13], and Li et al. [14] considered the oscillatory behavior of the third-order dynamic equation

$$
\left(\left(x^{\Delta \Delta}(t)\right)^{\gamma}\right)^{\Delta}+p(t) x^{\gamma}(\tau(t))=0, \quad t \in \mathbb{T}, t \geq t_{0},
$$

where $\gamma>0$ is the ratio of positive odd integers, and proved the following results.

Theorem 1.1 Assume that $\int_{t_{0}}^{\infty} p(t)\left(h_{2}\left(\tau(t), t_{0}\right)\right)^{\gamma} \Delta t=\infty$ holds. Furthermore, suppose that there exists a positive function $\delta(t) \in C_{r d}^{1}(\mathbb{T}, \mathbb{R})$ such that

$$
\limsup _{t \rightarrow+\infty} \int_{t_{1}}^{t}\left[k p(s) \delta^{\sigma}(s) \zeta(s)-\frac{1}{(\gamma+1)^{\gamma+1}} \frac{\left(\left(\delta^{\Delta}(s)\right)_{+}\right)^{\gamma+1}}{\left(\delta^{\sigma}(s) \alpha(s)\right)^{\gamma}}\right] \Delta s=\infty
$$

where $0<k<1, \zeta(t)=\left(\frac{h_{2}\left(\tau(t), t_{0}\right)}{\sigma(t)}\right)^{\gamma}$. Then every solution $x(t)$ of Eq. (1.6) is either oscillatory or $\lim _{t \rightarrow \infty} x(t)=0$.

Yang [15] studied the oscillatory and asymptotic behavior of third-order nonlinear variable delay dynamic equations

$$
r(t) \phi\left(\left[a(t) y^{\Delta}(t)\right]^{\Delta}\right)^{\Delta}+P(t) F(\phi(x(\delta(t))))=0, \quad t \in \mathbb{T}, t \geq t_{0},
$$

where $y(t)=x(t)+B(t) g(x(\tau(t))), \phi(u)=|u|^{\lambda-1} u, \lambda>0$ is the ratio of positive odd integers, and got the following results.

Theorem 1.2 If there exists a function $\delta(t) \in C_{r d}^{1}(\mathbb{T}, \mathbb{R})$ such that

$$
\limsup _{t \rightarrow+\infty} \int_{t_{1}}^{t}\left[L \varphi(s) P(s) \Psi^{\lambda}(s)-\frac{1}{(\lambda+1)^{\lambda+1}} \frac{\left[\left|\varphi^{\Delta}(s)\right| r(\sigma(s))\right]^{\lambda+1}}{[r(s) \varphi(s)]^{\lambda}}\right] \Delta s=\infty
$$

where $\Psi(t)=\frac{k[1-\beta B(\delta(t))] h_{2}\left(\delta(t), t_{0}\right)}{2 a(\delta(t)) \sigma(t)}$, then every solution $x(t)$ of $E q$. (1.8) is either oscillatory or $\lim _{t \rightarrow \infty} x(t)=0$.

Grace [16] was concerned with new oscillation criteria for third-order nonlinear difference equations with a nonlinear non-positive neutral term of the form

$$
\Delta\left(\left(a(t)\left(\Delta^{2}\left(x(t)-p(t) x^{\alpha}(t-k)\right)\right)\right)^{\gamma}\right)+q(t) x^{\beta}(t-m+1)=0,
$$

where $\alpha, \gamma$, and $\beta$ are the ratios of positive odd integers.

Candan [17] was concerned with oscillation of the third-order nonlinear neutral dynamic equation

$$
\left[r_{2}(t)\left(\left(r_{1}(t)(y(t)+p(t) y(\tau(t)))^{\Delta}\right)^{\Delta}\right)^{\gamma}\right]^{\Delta}+f(t, y(\delta(t)))=0, \quad t \in\left[t_{0}, \infty\right]_{\mathbb{T}},
$$

where $\gamma \geq 1$ is a ratio of odd positive integers.

It would also be interesting to establish sufficient conditions for the oscillation and asymptotic behavior of solutions to Eq. (1.1) for the other ranges of the neutral coefficient $p(t)$ such as (1.10). For this research issue, see, e.g., the papers $[18,19]$ for more details. 
It is not difficult to see that the results on the oscillation of delay dynamic (differential/difference) equations with sublinear neutral terms are mainly concentrated in the second order (see [3, 6-8] and the references cited therein), but the results of the third order are relatively lower $[16,20]$. Numerous researchers have studied the special case of Eq. (1.1), see, e.g., Ref [9-30]. Inspired by the above papers, in this paper, by employing the Riccati transformation and some integral inequality, we present sufficient conditions to ensure that every solution of Eq. (1.1) is oscillatory or asymptotically converges to zero. Our results improve and generalize the results of the papers $[13,15]$.

\section{Preliminaries}

Before proving the main theorems, we need some useful lemmas which will be used later. In [1], p. 190, Taylor's monomials $h_{n}(t, s)_{n=0}^{\infty}$ are defined recursively by

$$
h_{0}(t, s)=1, h_{n+1}(t, s)=\int_{s}^{t} h_{n}(\tau, s) \Delta \tau, \quad t, s \in \mathbb{T}, n \geq 1 .
$$

It follows from [1] that $h_{1}(t, s)=t-s$ for any time-scale, but simple formulas in general do not hold for $n \geq 2$.

Lemma 2.1 ([10]) If u satisfies $u(t)>0, u^{\Delta}(t)>0, u^{\Delta \Delta}(t)>0, u^{\Delta \Delta \Delta}(t)<0, t \in\left[t_{0}, \infty\right)_{\mathbb{T}}$, then

$$
\liminf _{t \rightarrow \infty} \frac{t u(t)}{h_{2}\left(t, t_{0}\right) u^{\Delta}(t)} \geq 1
$$

Lemma 2.2 ([10]) Set the following conditions to be established:

(1) $u \in C_{r d}^{2}(\mathbb{I}, \mathbb{R})$, where $\mathbb{I}=\left[t_{0}, \infty\right)_{\mathbb{T}}$,

(2) $u(t)>0, u^{\Delta}(t)>0$, and $u^{\Delta \Delta}(t)<0, t \in \mathbb{I}$.

Then, for any $k \in(0,1)$, there exists $t_{1} \in \mathbb{I}$ such that, while $t \in \mathbb{I}$ and $t \geq t_{1}$,

$$
u(\sigma(t)) \leq \frac{\sigma(t)}{k \tau(t)} u(\tau(t))
$$

Lemma 2.3 Keller's chain rule

$$
\left(z^{\beta}(t)\right)^{\Delta} \geq \begin{cases}\beta \int_{0}^{1}(z(\sigma(t)))^{\beta-1} z^{\Delta}(t) d h=\beta(z(\sigma(t)))^{\beta-1} z^{\Delta}(t), & 0<\beta \leq 1, \\ \beta \int_{0}^{1}(z(t))^{\beta-1} z^{\Delta}(t) d h=\beta(z(t))^{\beta-1} z^{\Delta}(t), & \beta>1,\end{cases}
$$

where $z(t)$ is $\Delta$-differentiable and eventually positive or eventually negative.

Its proof is not difficult from the monograph [1, Theorem 2.57], and so it is omitted here.

Lemma 2.4 Assume that $A \geq 0, B>0$, and $\gamma>0$ are arbitrary real numbers. Then, for all $X \geq 0$,

$$
A X-B X^{\frac{1+\gamma}{\gamma}} \leq \frac{\gamma^{\gamma}}{(\gamma+1)^{\gamma+1}} \cdot \frac{A^{\gamma+1}}{B^{\gamma}} .
$$


Proof Let $g(X)=A X-B X^{\frac{\gamma+1}{\gamma}}, X \in[0, \infty), A \geq 0, B>0$, and $\gamma>0$ be arbitrary real numbers and

$$
g^{\prime}(X)=A-\frac{\gamma+1}{\gamma} B X^{\frac{1}{\gamma}}=0 .
$$

We can obtain $X^{*}=\left(\frac{A \gamma}{B(\gamma+1)}\right)^{\gamma}$, the unique solution to (2.5). It is clear that $g^{\prime}(X)>0$ for all $X<X^{*}$ and $g^{\prime}(X)<0$ for all $X>X^{*}$. Then $g(X)$ attains its maximum value on $[0, \infty)$ at $X^{*}$, and

$$
\max _{X \in[0, \infty)} g(X)=g\left(X^{*}\right)=\frac{\gamma^{\gamma}}{(\gamma+1)^{\gamma+1}} \frac{A^{\gamma+1}}{B^{\gamma}} .
$$

Thus (2.4) holds, this completes the proof of the lemma.

It is obvious that Lemma 2.4 is an improvement of Lemma 2.3 in [31] about the constant $\gamma>0$, which must be a ratio of two positive odd integers is no longer suitable for Eq. (1.1).

\section{Main results}

Theorem 3.1 Assume that (1.2) holds. If there exists a positive function $\eta(t) \in C_{r d}^{1}(\mathbb{T}, \mathbb{R})$ such that, for any constants $L \in(0,1), M \in(0,1]$ (if $\alpha=1$ have $M=1$ ), and sufficiently large $t_{1} \geq t_{0}>0$,

$$
\limsup _{t \rightarrow+\infty} \int_{t_{1}}^{t}\left[K(s)-\frac{1}{(\gamma+1)^{\gamma+1}} \frac{\left(\left(\eta^{\Delta}(s)\right)_{+} b(\sigma(s))\right)^{\gamma+1}}{(\eta(s) b(s))^{\gamma}}\right] \Delta s=\infty
$$

and

$$
\int_{t_{1}}^{\infty} \frac{1}{a(v)} \int_{v}^{\infty} b^{-\frac{1}{\gamma}}(u)\left(\int_{u}^{\infty} q(s) \Delta s\right)^{\frac{1}{\gamma}} \Delta u \Delta v=\infty
$$

hold, where

$$
K(t)=L Q(t)\left(\frac{h_{2}\left(\tau(t), t_{0}\right)}{\sigma(t) a(\tau(t))}\right)^{\gamma}, \quad Q(t)=\eta(t) q(t)\left(1-\frac{p(\tau(t))}{M^{1-\alpha}}\right)^{\beta}
$$

then every solution $x(t)$ of Eq. (1.1) is either oscillatory or $\lim _{t \rightarrow \infty} x(t)=0$.

Proof Assume to the contrary that $x(t)$ is an eventually positive solution of Eq. (1.1), that is, there exists $t_{1}>t_{0}$ such that $x(t)>0, x(\tau(t))>0, x(\delta(t))>0$ for all $t \in\left[t_{1}, \infty\right)_{\mathbb{T}}, t_{1} \in\left[t_{0}, \infty\right)_{\mathbb{T}}$. Then $z(t)$ has two possible cases:

(I) $z(t)>0, z^{\Delta}(t)>0,\left(a(t) z^{\Delta}(t)\right)^{\Delta}>0,\left(b(t)\left(\left(a(t) z^{\Delta}(t)\right)^{\Delta}\right)^{\gamma}\right)^{\Delta}<0$;

(II) $z(t)>0, z^{\Delta}(t)<0,\left(a(t) z^{\Delta}(t)\right)^{\Delta}>0,\left(b(t)\left(\left(a(t) z^{\Delta}(t)\right)^{\Delta}\right)^{\gamma}\right)^{\Delta}<0$.

If case (I) holds, then $z(t)>0, z(\tau(t))>0, z(\delta(t))>0$ for all $t>t_{1}$. Furthermore, in view of the fact that $z(t)$ is monotonically increasing on $\left[t_{1}, \infty\right)_{\mathbb{T}}$, there exists a positive constant $M_{0}$ such that $z(t)>M_{0}$, where $M_{0}=\min \left\{z\left(t_{1}\right), 1\right\} \in(0,1]$. According to the definition of $z(t)$, we can easily get

$$
x(t) \geq\left(1-\frac{p(t)}{M_{0}^{1-\alpha}}\right) z(t), \quad t \geq t_{1} .
$$


From Eq. (1.1) and the above inequality, we have

$$
\left(b(t)\left(\left(a(t) z^{\Delta}(t)\right)^{\Delta}\right)^{\gamma}\right)^{\Delta}+q(t)\left(1-\frac{p(\tau(t))}{M_{0}^{1-\alpha}}\right)^{\beta} z^{\beta}(\tau(t)) \leq 0, \quad t \geq t_{1} .
$$

Define the generalized Riccati transformation

$$
\omega(t)=\eta(t) \frac{b(t)\left(\left(a(t) z^{\Delta}(t)\right)^{\Delta}\right)^{\gamma}}{\left(a(t) z^{\Delta}(t)\right)^{\gamma}}, \quad t \geq t_{1} .
$$

Letting $u(t)=a(t) z^{\Delta}(t)$, the above formula can be abbreviated as

$$
\omega(t)=\eta(t) \frac{b(t)\left(u^{\Delta}(t)\right)^{\gamma}}{u^{\gamma}(t)}, \quad t \geq t_{1}
$$

Obviously $\omega(t)>0$ for $t \geq t_{1}$, and by the product rule and the quotient rule on time scales, we have

$$
\begin{aligned}
\omega^{\Delta}(t) & =\eta^{\Delta}(t) \frac{b(\sigma(t))\left(u^{\Delta}(\sigma(t))\right)^{\gamma}}{u^{\gamma}(\sigma(t))}+\eta(t)\left(\frac{b(t)\left(u^{\Delta}(t)\right)^{\gamma}}{u^{\gamma}(t)}\right)^{\Delta} \\
& =\frac{\eta^{\Delta}(t)}{\eta(\sigma(t))} \omega(\sigma(t))+\eta(t) \frac{\left(b(t)\left(u^{\Delta}(t)\right)^{\gamma}\right)^{\Delta}}{u^{\gamma}(\sigma(t))}-\eta(t) \frac{b(t)\left(u^{\Delta}(t)\right)^{\gamma}\left(u^{\gamma}(t)\right)^{\Delta}}{u^{\gamma}(t) u^{\gamma}(\sigma(t))}, \quad t \geq t_{1} .
\end{aligned}
$$

From (3.4), (3.6), and the definition of $\omega(t)$, we can obtain

$$
\omega^{\Delta}(t) \leq-\frac{Q(t) z^{\beta}(\tau(t))}{u^{\gamma}(\sigma(t))}+\frac{\left(\eta^{\Delta}(t)\right)_{+}}{\eta(\sigma(t))} \omega(\sigma(t))-\eta(t) \frac{b(t)\left(u^{\Delta}(t)\right)^{\gamma}\left(u^{\gamma}(t)\right)^{\Delta}}{u^{\gamma}(t) u^{\gamma}(\sigma(t))}, \quad t \geq t_{1},
$$

where

$$
\left(\eta^{\Delta}(t)\right)_{+}=\max \left\{0, \eta^{\Delta}(t)\right\}, \quad Q(t)=\eta(t) q(t)\left(1-\frac{p(\tau(t))}{M_{0}^{1-\alpha}}\right)^{\beta}
$$

In view of the fact that $\left(b(t)\left(u^{\Delta}(t)\right)^{\gamma}\right)^{\Delta} \leq 0$, we have

$$
\left(b(t)\left(u^{\Delta}(t)\right)^{\gamma}\right)^{\Delta}=b^{\Delta}(t)\left(u^{\Delta}(\sigma(t))\right)^{\gamma}+\gamma b(t)\left(u^{\Delta}(t)\right)^{\gamma-1} u^{\Delta \Delta}(t) \leq 0, \quad t \geq t_{1} .
$$

Also $b^{\Delta}(t)>0$, then, it is not hard for us to get $u^{\Delta \Delta}(t)<0$ for $t \geq t_{1}$. Further, we have

$$
u(t) \leq u(\sigma(t)) \quad \text { and } \quad u^{\Delta}(t) \geq u^{\Delta}(\sigma(t)), \quad t \geq t_{1} .
$$

If $\gamma>1$, by (3.9), we can get

$$
\begin{aligned}
\omega^{\Delta}(t) & \leq-\frac{Q(t) z^{\beta}(\tau(t))}{u^{\gamma}(\sigma(t))}+\frac{\left(\eta^{\Delta}(t)\right)_{+}}{\eta(\sigma(t))} \omega(\sigma(t))-\gamma \eta(t) \frac{b(t)\left(u^{\Delta}(t)\right)^{\gamma} u^{\Delta}(t)}{u^{\gamma}(\sigma(t)) u(t)} \\
& \leq-\frac{Q(t) z^{\beta}(\tau(t))}{u^{\gamma}(\sigma(t))}+\frac{\left(\eta^{\Delta}(t)\right)_{+}}{\eta(\sigma(t))} \omega(\sigma(t))-\gamma \eta(t) b(t) \frac{\left(u^{\Delta}(t)\right)^{\gamma+1}}{(u(\sigma(t)))^{\gamma+1}} \\
& \leq-\frac{Q(t) z^{\beta}(\tau(t))}{u^{\gamma}(\sigma(t))}+\frac{\left(\eta^{\Delta}(t)\right)_{+}}{\eta(\sigma(t))} \omega(\sigma(t))-\frac{\gamma \eta(t) b(t)}{(b(\sigma(t)) \eta(\sigma(t)))^{\frac{\gamma+1}{\gamma}}} \omega^{\frac{\gamma+1}{\gamma}}(\sigma(t))
\end{aligned}
$$




$$
=-Q(t) z^{\beta-\gamma}(\tau(t)) \cdot\left(\frac{z(\tau(t))}{u(\sigma(t))}\right)^{\gamma}+P(t), \quad t \geq t_{1},
$$

where

$$
P(t)=\frac{\left(\eta^{\Delta}(t)\right)_{+}}{\eta(\sigma(t))} \omega(\sigma(t))-\frac{\gamma \eta(t) b(t)}{(b(\sigma(t)) \eta(\sigma(t)))^{\frac{\gamma+1}{\gamma}}} \omega^{\frac{\gamma+1}{\gamma}}(\sigma(t)) .
$$

Since $z(t)$ is monotonically increasing on $\left[t_{1}, \infty\right)_{\mathbb{T}}$, we get $z\left(t_{1}\right)<z(\tau(t))$ for $t \geq t_{1}$. Also, since the definition of $M_{0}$ and $\beta \geq \gamma$, we have

$$
z^{\beta-\gamma}(\tau(t)) \geq z^{\beta-\gamma}\left(t_{1}\right) \geq M_{0}^{\beta-\gamma}, \quad t \geq t_{1}
$$

and

$$
\omega^{\Delta}(t) \leq-M_{0}^{\beta-\gamma} Q(t) \cdot\left(\frac{z(\tau(t))}{u(\sigma(t))}\right)^{\gamma}+P(t), \quad t \geq t_{1} .
$$

In addition, since (3.8), we can easily get $u(t)$, which satisfies Lemma 2.2, then there exists a constant $k \in(0,1)$ such that

$$
u(\sigma(t)) \leq \frac{\sigma(t) u(\tau(t))}{k \tau(t)}, \quad t \geq t_{1}
$$

When letting $v(t)=\int_{t_{1}}^{t} a(s) z^{\Delta}(s) \Delta s$, we can see that it satisfies Lemma 2.1. So there exists some $t_{l} \in\left[t_{1}, \infty\right)_{\mathbb{T}}$ such that

$$
\frac{v(t)}{v^{\Delta}(t)} \geq l \frac{h_{2}\left(t, t_{0}\right)}{t}, \quad t \geq t_{1}
$$

for any $t \in\left[t_{1}, \infty\right)_{\mathbb{T}}$ and for any $l \in(0,1)$. Meanwhile, we can easily deduce $a(t) z(t)>$ $\int_{t_{1}}^{t} a(s) z^{\Delta}(s) \Delta s$, so

$$
\frac{z(t)}{z^{\Delta}(t)}=\frac{a(t) z(t)}{a(t) z^{\Delta}(t)} \geq \frac{\int_{t_{1}}^{t} a(s) z^{\Delta}(s) \Delta s}{a(t) z^{\Delta}(t)} \geq l \frac{h_{2}\left(t, t_{0}\right)}{t}, \quad t \geq t_{1},
$$

and then

$$
\frac{z(\tau(t))}{u(\sigma(t))}=\frac{z(\tau(t))}{a(\sigma(t)) z^{\Delta}(\sigma(t))}=\frac{1}{a(\sigma(t))} \frac{z(\tau(t))}{z^{\Delta}(\tau(t))} \frac{z^{\Delta}(\tau(t))}{z^{\Delta}(\sigma(t))} \leq \frac{k \operatorname{lh}_{2}\left(\tau(t), t_{0}\right)}{\sigma(t) a(\tau(t))}
$$

for all $t \geq t_{1}$. Substituting the above formula into (3.10), we conclude that

$$
\omega^{\Delta}(t) \leq-L Q(t) \cdot\left(\frac{h_{2}\left(\tau(t), t_{0}\right)}{\sigma(t) a(\tau(t))}\right)^{\gamma}+P(t), \quad t \geq t_{1}
$$

where $L=M_{0}^{\beta-\gamma}(k l)^{\gamma} \in(0,1)$. Apply inequality (2.4) to $P(t)$ with

$$
A=\frac{\left(\eta^{\Delta}(t)\right)_{+}}{\eta(\sigma(t))}, \quad B=\frac{\gamma \eta(t) b(t)}{(b(\sigma(t)) \eta(\sigma(t)))^{\frac{\gamma+1}{\gamma}}} \quad \text { and } \quad X=\omega(\sigma(t)) \text {, }
$$


which yields

$$
P(t) \leq \frac{1}{(\gamma+1)^{\gamma+1}} \frac{\left(\left(\eta^{\Delta}(t)\right)_{+} b(\sigma(t))\right)^{\gamma+1}}{(\eta(t) b(t))^{\gamma}}, \quad t \geq t_{1} .
$$

Substituting the above formula into (3.15), we have

$$
\omega^{\Delta}(t) \leq-L Q(t) \cdot\left(\frac{h_{2}\left(\tau(t), t_{0}\right)}{\sigma(t) a(\tau(t))}\right)^{\gamma}+\frac{1}{(\gamma+1)^{\gamma+1}} \frac{\left(\left(\eta^{\Delta}(t)\right)_{+} b(\sigma(t))\right)^{\gamma+1}}{(\eta(t) b(t))^{\gamma}}
$$

for all $t \geq t_{1}$. Similarly, if $0<\gamma<1$, it is easy for us to get (3.17). From inequality (3.17), we have

$$
L Q(t) \cdot\left(\frac{h_{2}\left(\tau(t), t_{0}\right)}{\sigma(t) a(\tau(t))}\right)^{\gamma}-\frac{1}{(\gamma+1)^{\gamma+1}} \frac{\left(\left(\eta^{\Delta}(t)\right)_{+} b(\sigma(t))\right)^{\gamma+1}}{(\eta(t) b(t))^{\gamma}} \leq-\omega^{\Delta}(t), \quad t \geq t_{1} .
$$

Integrating the above inequality from sufficiently large $t_{1}$ to $t \geq t_{1}$, we have

$$
\begin{aligned}
& \int_{t_{1}}^{t} L Q(s) \cdot\left(\frac{h_{2}\left(\tau(s), t_{0}\right)}{\sigma(s) a(\tau(s))}\right)^{\gamma}-\frac{1}{(\gamma+1)^{\gamma+1}} \frac{\left(\left(\eta^{\Delta}(s)\right)_{+} b(\sigma(s))\right)^{\gamma+1}}{(\eta(s) b(s))^{\gamma}} \Delta s \\
& \quad \leq-\int_{t_{1}}^{t} \omega^{\Delta}(s) \Delta s=\omega\left(t_{1}\right)-\omega(t)<\omega\left(t_{1}\right) .
\end{aligned}
$$

Take the limsup on both sides of the above inequality as $t \rightarrow \infty$, which contradicts (3.1).

If case (II) holds, by $z(t)>0$ and $z^{\Delta}(t)<0$, we know that $z(t)$ is decreasing and $\lim _{t \rightarrow+\infty} z(t)=l \geq 0$. We assert that $l=0$. If not, then $l>0$. By the definition of limit, we pick $0<\varepsilon<l^{2-\alpha}(1-p) /(\alpha p)$ and large enough $t_{2} \in\left[t_{1}, \infty\right)_{\mathbb{T}}$ such that $l \leq z(t) \leq l+\varepsilon$ for all $t \in\left[t_{2}, \infty\right)_{\mathbb{T}}$. So that

$$
x(t)=z(t)-p(t) x^{\alpha}(\delta(t))>l-p(l+\varepsilon)^{\alpha}>k(l+\varepsilon)>k z(t),
$$

where $k=\frac{l-p(l+\varepsilon)^{\alpha}}{l+\varepsilon}>0$, and thus

$$
x(\tau(t))>k z(\tau(t)), \quad t \geq t_{2} .
$$

Substituting it into Eq. (1.1), we obtain

$$
\left(b(t)\left(\left(a(t) z^{\Delta}(t)\right)^{\Delta}\right)^{\gamma}\right)^{\Delta} \leq-k^{\beta} q(t) z^{\beta}(\tau(t)), \quad t \geq t_{2} .
$$

Integrating the above formula from sufficiently large $t \geq t_{2}$ to $\infty$ and noting that $z^{\beta}(\tau(t)) \geq$ $l^{\beta}$, we have

$$
b(t)\left(\left(a(t) z^{\Delta}(t)\right)^{\Delta}\right)^{\gamma} \geq(k l)^{\beta} \int_{t}^{\infty} q(s) \Delta s, \quad t \geq t_{2},
$$

and then

$$
\left(a(t) z^{\Delta}(t)\right)^{\Delta} \geq\left(\frac{(k l)^{\beta} \int_{t_{2}}^{\infty} q(s) \Delta s}{b(t)}\right)^{\frac{1}{\gamma}}, \quad t \geq t_{2} .
$$


Integrating the above inequality from sufficiently large $t$ to $\infty$, we have

$$
-z^{\Delta}(t) \geq(k l)^{\frac{\beta}{\gamma}} \frac{1}{a(t)} \int_{t}^{\infty} b^{-\frac{1}{\gamma}}(u)\left(\int_{u}^{\infty} q(s) \Delta s\right)^{\frac{1}{\gamma}} \Delta u, \quad t \geq t_{2} .
$$

Integrating the last inequality again from $t$ to $\infty$, we can obtain

$$
z\left(t_{1}\right) \geq(k l)^{\frac{\beta}{\gamma}} \int_{t_{1}}^{\infty} \frac{1}{a(v)} \int_{v}^{\infty} b^{-\frac{1}{\gamma}}(u)\left(\int_{u}^{\infty} q(s) \Delta s\right)^{\frac{1}{\gamma}} \Delta u \Delta v, \quad t \geq t_{2},
$$

which contradicts (3.2), hence $l=0$. The proof is completed.

Corollary 3.2 The conclusion of Theorem 3.1 remains intact if assumption (3.1) is replaced with two conditions

$$
\limsup _{t \rightarrow+\infty} \int_{t_{1}}^{t} Q(s) \cdot\left(\frac{h_{2}\left(\tau(s), t_{0}\right)}{\sigma(s) a(\tau(s))}\right)^{\gamma} \Delta s=\infty
$$

and

$$
\liminf _{t \rightarrow+\infty} \int_{t_{1}}^{t} \frac{\left(\left(\eta^{\Delta}(s)\right)_{+} b(\sigma(s))\right)^{\gamma+1}}{(\eta(s) b(s))^{\gamma}} \Delta s<\infty
$$

Remark 3.3 Obviously, the result of Theorem 3.1 is more extensive. When the corresponding coefficients of Eq. (1.1) are equal to those of Eq. (1.6) and Eq. (1.8), Eq. (1.1) can be simplified into Eq. (1.6) or (1.8). In this case, Theorem 1.1 or Theorem 1.2 can be completely included.

Remark 3.4 From Theorem 3.1, we can obtain different conditions for oscillation of Eq. (1.1) with different choices of $\eta(t)$. For example, $\eta(t)=t$ or $\eta(t)=1$. Now, letting us consider $\eta(t)=1$, we have the following corollary.

Corollary 3.5 Assume that (1.2) holds. If, for sufficiently large $t_{1} \geq t_{0}>0$,

$$
\limsup _{t \rightarrow+\infty} \int_{t_{1}}^{t} Q_{1}(s) \cdot\left(\frac{h_{2}\left(\tau(s), t_{0}\right)}{\sigma(s) a(\tau(s))}\right)^{\gamma} \Delta s=\infty
$$

holds, where $Q_{1}(t)=q(t)\left(1-\frac{p(\tau(t))}{\left.M^{1-\alpha}\right)^{\beta}}\right.$, and $M$ is the same as in Theorem 3.1, then every solution $x(t)$ of Eq. (1.1) is either oscillatory or $\lim _{t \rightarrow \infty} x(t)=0$.

The following theorem gives the Philos-type oscillation criterion for Eq. (1.1). First, let us introduce now the class of functions $\mathfrak{H}$ which will be extensively used in the sequel.

Let $\mathbb{D}=:\left\{(t, s): t \geq s \geq t_{0}\right\}$ and $\mathbb{D}_{0}=:\left\{(t, s): t>s \geq t_{0}\right\}$. The function $H \in C(\mathbb{D}, \mathbb{R})$ is said to belong to the class $\mathfrak{H}$ if it satisfies the following conditions:

$$
\begin{aligned}
& \left(H_{1}\right) H(t, t)=0, t \in \mathbb{D}, H(t, s)>0, t \in \mathbb{D}_{0} ; \\
& \left(H_{2}\right) H^{\Delta s}(t, t)=0, t \in \mathbb{D}, H^{\Delta s}(t, s) \leq 0, t \in \mathbb{D}_{0} .
\end{aligned}
$$


Theorem 3.6 Assume that (1.2) holds. Furthermore, suppose that there exist two positive functions $\eta(t) \in C_{r d}^{1}(\mathbb{T}, \mathbb{R})$ and $h(t, s) \in C_{r d}(\mathbb{D}, \mathbb{R})$ satisfying conditions $\left(H_{1}\right),\left(H_{2}\right)$, and

$$
\left(H_{3}\right) \quad H^{\Delta_{s}}(\sigma(t), \sigma(s))+H(\sigma(t), \sigma(s)) \frac{\left(\eta^{\Delta}(t)\right)_{+}}{\eta(t)}=-\frac{h(t, s)}{\eta(t)} H^{\frac{\gamma}{\gamma+1}}(\sigma(t), \sigma(s)) .
$$

If, for sufficiently large $t_{1} \geq t_{0}>0$,

$$
\limsup _{t \rightarrow+\infty} \frac{1}{H\left(\sigma(t), \sigma\left(t_{1}\right)\right)} \int_{t_{1}}^{t}\left[K_{1}(s)-\frac{\left(h_{-}(t, s) b(\sigma(s))\right)^{\gamma+1}}{(\gamma+1)^{\gamma+1}(\eta(s) b(s))^{\gamma}}\right] \Delta s=\infty
$$

holds, where $K_{1}(t)=H(\sigma(t), \sigma(s)) K(s)$ and $K(t)$ is defined by (3.1), then every solution $x(t)$ of Eq. (1.1) is either oscillatory or $\lim _{t \rightarrow \infty} x(t)=0$.

Proof Assume to the contrary that $x(t)$ is an eventually positive solution of Eq. (1.1), that is, there exists $t_{1}>t_{0}$ such that $x(t)>0, x(\tau(t))>0, x(\delta(t))>0$ for all $t \in\left[t_{1}, \infty\right)_{\mathbb{T}}$. Then $z(t)$ satisfies case (I) or (II). If case (I) holds, we process as in the proof of Theorem 3.1 and get (3.15) as follows:

$$
K(t)=L Q(t)\left(\frac{h_{2}\left(\tau(t), t_{0}\right)}{\sigma(t) a(\tau(t))}\right)^{\gamma} \leq-\omega^{\Delta}(t)+P(t), \quad t \geq t_{1} .
$$

Multiplying both sides of (3.23), with $t$ replaced with $s$, by $H(\sigma(t), \sigma(s))$, integrating with respect to $s$ from $t_{1}$ to $t \geq t_{1}$, we have

$$
\begin{aligned}
& \int_{t_{1}}^{t} H(\sigma(t), \sigma(s)) K(s) \Delta s \\
& \quad \leq-\int_{t_{1}}^{t} H(\sigma(t), \sigma(s)) \omega^{\Delta}(s) \Delta s+\int_{t_{1}}^{t} H(\sigma(t), \sigma(s)) P(s) \Delta s .
\end{aligned}
$$

Integrating by parts, we obtain

$$
\begin{aligned}
& \int_{t_{1}}^{t} H(\sigma(t), \sigma(s)) \omega^{\Delta}(s) \Delta s \\
& \quad=-H\left(\sigma(t), \sigma\left(t_{1}\right)\right) \omega\left(t_{1}\right)-\int_{t_{1}}^{t} H^{\Delta_{s}}(\sigma(t), \sigma(s)) \omega(\sigma(s)) \Delta s .
\end{aligned}
$$

Using conditions $\left(H_{1}\right)-\left(H_{3}\right)$ and inequality (2.4), we deduce that

$$
\begin{aligned}
& \int_{t_{1}}^{t} H(\sigma(t), \sigma(s)) K(s) \Delta s \\
& \quad \leq H\left(\sigma(t), \sigma\left(t_{1}\right)\right) \omega\left(t_{1}\right)+\int_{t_{1}}^{t} \frac{h_{-}(t, s)}{\eta(\sigma(s))} H^{\frac{\gamma}{\gamma+1}}(\sigma(t), \sigma(s)) \omega(\sigma(s)) \Delta s \\
& \quad-\int_{t_{1}}^{t} H(\sigma(t), \sigma(s)) \frac{\gamma \eta(t) b(t)}{(b(\sigma(t)) \eta(\sigma(t)))^{\frac{\gamma+1}{\gamma}}} \omega^{\frac{\gamma+1}{\gamma}}(t) \Delta s \\
& \leq H\left(\sigma(t), \sigma\left(t_{1}\right)\right) \omega\left(t_{1}\right)+\frac{1}{(\gamma+1)^{\gamma+1}} \cdot \frac{\left(h_{-}(t, s) b(\sigma(s))\right)^{\gamma+1}}{(\eta(s) b(s))^{\gamma}} \Delta s, \quad t \geq t_{1},
\end{aligned}
$$


this implies that

$$
\begin{aligned}
& \frac{1}{H\left(\sigma(t), \sigma\left(t_{1}\right)\right)} \int_{t_{1}}^{t}\left[H(\sigma(t), \sigma(s)) K(s)-\frac{1}{(\gamma+1)^{\gamma+1}} \cdot \frac{\left(h_{-}(t, s) b(\sigma(s))\right)^{\gamma+1}}{(\eta(s) b(s))^{\gamma}}\right] \Delta s \\
& \quad \leq \omega\left(t_{1}\right), \quad t \geq t_{1},
\end{aligned}
$$

which contradicts condition (3.22).

If case (II) holds, then clearly $\lim _{t \rightarrow \infty} x(t)=0$. The proof is completed.

Remark 3.7 From Theorem 3.6, we can obtain different conditions for oscillation of all solutions of Eq. (1.1) with different choices of $\eta(t)$ and $H(t, s)$. For example, $H(t, s)=(t-s)^{m}$ or $H(t, s)=\left(\ln \frac{t+1}{s+1}\right)^{m}$. Now, let us consider the function $H(t, s)$ defined by

$$
H(t, s)=(t-s)^{m}, \quad m \geq 1, t \geq s \in\left[t_{0}, \infty\right)_{\mathbb{T}} .
$$

Hence we have the following Kamenev-type oscillation criterion for Eq. (1.1).

Corollary 3.8 Assume that (1.2) holds. Furthermore, suppose that there exist a positive function $\eta(t) \in C_{r d}^{1}(\mathbb{T}, \mathbb{R})$ and a constant $m \geq 1$ such that, for all sufficiently large $t_{1} \geq t_{0}>0$,

$$
\limsup _{t \rightarrow+\infty} \frac{1}{t^{m}} \int_{t_{1}}^{t}\left[(t-s)^{m} K(s)-\frac{1}{(\gamma+1)^{\gamma+1}} \cdot\left(\frac{h_{-}(t, s) b(\sigma(s))}{\eta(s) b(s)}\right)^{\gamma+1}\right] \Delta s=\infty
$$

holds, where $K(t)$ is defined by (3.1). Then every solution $x(t)$ of Eq. (1.1) is either oscillatory or $\lim _{t \rightarrow \infty} x(t)=0$.

Corollary 3.9 Assume that (1.2) holds. Furthermore, suppose that there exist two positive functions $\eta(t) \in C_{r d}^{1}(\mathbb{T}, \mathbb{R})$ and $r(t, s) \in C_{r d}(\mathbb{D}, \mathbb{R})$ satisfying conditions $\left(H_{1}\right),\left(H_{2}\right)$, and

$$
\left(H_{4}\right) \quad H^{\Delta_{s}}(\sigma(t), \sigma(s))+H(\sigma(t), \sigma(s)) \frac{\left(\eta^{\Delta}(t)\right)_{+}}{\eta(t)}=-\frac{r(t, s)}{\eta(t)} \sqrt{H(\sigma(t), \sigma(s))} .
$$

If, for all sufficiently large $t_{1} \geq t_{0}>0$,

$$
\limsup _{t \rightarrow+\infty} \frac{1}{H\left(\sigma(t), \sigma\left(t_{1}\right)\right)} \int_{t_{1}}^{t}\left[K_{1}(s)-\frac{H^{\frac{1-\gamma}{2}}(\sigma(t), \sigma(s))\left(r_{-}(t, s) b(\sigma(s))\right)^{\gamma+1}}{(\gamma+1)^{\gamma+1}(\eta(s) b(s))^{\gamma}}\right] \Delta s=\infty
$$

holds, where $K_{1}(t)$ is defined by (3.22), then every solution $x(t)$ of Eq. (1.1) is either oscillatory or $\lim _{t \rightarrow \infty} x(t)=0$.

\section{Application}

In this section, we give an example to illustrate our main results of this paper.

Example 4.1 Consider a third-order neutral dynamic equation

$$
\left(t^{3}\left(\left(e^{-t}\left(x(t)+\frac{1}{2 t} x^{\frac{1}{3}}(t-1)\right)^{\Delta}\right)^{\Delta}\right)^{3}\right)^{\Delta}+\lambda x^{5}\left(\frac{t}{2}\right)=0, \quad t \in[1, \infty)_{\mathbb{T}},
$$


where $\lambda>0$ is a constant.

Here,

$$
\begin{array}{lll}
\alpha=\frac{1}{3}, \quad \gamma=3, \quad \beta=5, & b(t)=t^{3}, \quad a(t)=e^{-t}, \quad p(t)=\frac{1}{2 t}, \\
q(t)=\lambda, \quad \delta(t)=t-1<t, \quad \tau(t)=\frac{t}{2}<t .
\end{array}
$$

According to [1], when $\mathbb{T}=\overline{2^{Z}}$,

$$
\sigma(t)=2 t, \quad h_{2}(t, s)=\frac{(t-s)(t-2 s)}{3} .
$$

Letting $\eta(t)=1$, it is clear that conditions $\left(A_{1}\right)-\left(A_{4}\right)$ and (1.2) hold. Next, we will prove that (3.21) is satisfied. It is the key to prove that the following

$$
\limsup _{t \rightarrow+\infty} \int_{t_{1}}^{t} Q_{1}(s) \cdot\left(\frac{h_{2}\left(\tau(s), t_{0}\right)}{\sigma(s) a(\tau(s))}\right)^{\gamma} \Delta s=\infty
$$

is true. Since, for any fixed $M \in(0,1]$, we have

$$
Q_{1}(t)=q(t)\left(1-\frac{p(\tau(t))}{M^{1-\alpha}}\right)^{\beta}=\lambda\left(1-\frac{\frac{1}{t}}{M^{\frac{2}{3}}}\right)^{5}=\lambda\left(1-M^{-\frac{2}{3}} t^{-1}\right)^{5} .
$$

And obviously, when $t>M^{-\frac{2}{3}}, 1-M^{-\frac{2}{3}} t^{-1} \geq \frac{1}{2}$ always holds. Therefore when $4=t_{1}>$ $2 M^{-\frac{2}{3}}=t_{0}$, we have

$$
h_{2}\left(\frac{t}{2}, 4\right)=\frac{\left(\frac{t}{2}-4\right)\left(\frac{t}{2}-8\right)}{3}=\frac{1}{12} t^{2}-2 t+\frac{32}{3} \geq 1 .
$$

Hence, from (4.3) and (4.4),

$$
\begin{aligned}
\int_{t_{1}}^{\infty} Q_{1}(s) \cdot\left(\frac{h_{2}\left(\tau(s), t_{0}\right)}{\sigma(s) a(\tau(s))}\right)^{\gamma} \Delta s & =\int_{t_{1}}^{\infty} \lambda\left(1-M^{-\frac{2}{3}} s^{-1}\right)^{5} \cdot\left(\frac{\frac{1}{12} s^{2}-2 s+\frac{32}{3}}{2 s \cdot e^{-\frac{s}{2}}}\right)^{3} \Delta s \\
& \geq \frac{\lambda}{2^{5}} \int_{t_{1}}^{\infty} e^{\frac{3 s}{2}} \Delta s=\infty
\end{aligned}
$$

holds and (4.2) is true. Thus, by Corollary 3.5, we know that every solution $x(t)$ of (4.1) is either oscillatory or $\lim _{t \rightarrow \infty} x(t)=0$.

Remark 4.2 As fairly noticed by the referees, it would be of interest to study Eq. (1.1) with nabla $(\nabla)$ derivative or mixed delta $(\Delta)$-nabla $(\nabla)$ derivatives.

\section{Conclusions}

In this paper, we consider the oscillation and asymptotic behavior of a class of third-order Emden-Fowler delay dynamic equations with sublinear neutral terms on time scales. Under the conditions of $\left(A_{1}\right)-\left(A_{4}\right)$, the above theorems and related corollaries are obtained, and the correctness of the conclusion is verified by an example. 
Acknowledgements

The authors would like to thank the referees for their useful suggestions.

\section{Funding}

This project is supported by the National Sciences Foundation of China (11701528 and 11647034).

\section{Availability of data and materials}

Data sharing not applicable to this article as no data sets were generated or analyzed during the current study.

\section{Competing interests}

The authors declare that they have no competing interests.

\section{Authors' contributions}

All authors contributed equally to this work. All authors read and approved the final manuscript.

\section{Author details}

'Department of Mathematics, Taiyuan Institute of Technology, Taiyuan, China. ${ }^{2}$ Department of Mathematics, North University of China, Taiyuan, China.

\section{Publisher's Note}

Springer Nature remains neutral with regard to jurisdictional claims in published maps and institutional affiliations.

\section{Received: 12 September 2020 Accepted: 1 January 2021 Published online: 19 January 2021}

\section{References}

1. Bohner, M., Peterson, A.: Dynamic Equations on Time Scales, An Introduction with Applications. Birkhäuer, Base (2001)

2. Bohner, M., Hassan, T.S., Li, T.: Fite-Hille-Wintner-type oscillation criteria for second-order half-linear dynamic equations with deviating arguments. Indag. Math. 29(2), 548-560 (2018)

3. Dzurina, J., Grace, S.R., Jadlovska, I., Li, T.: Oscillation criteria for second-order Emden-Fowler delay differential equations with a sublinear neutral term. Math. Nachr. 293(5), 910-922 (2020)

4. Li, T., Pintus, N., Viglialoro, G.: Properties of solutions to porous medium problems with different sources and boundary conditions. Z. Angew. Math. Phys. 70(3), 86 (2019)

5. Li, T., Rogovchenko, Y.V.: Oscillation criteria for second-order superlinear Emden-Fowler neutral differential equations. Monatshefte Math. 184(3), 489-500 (2017)

6. Agarwal, R.P., Bohner, M., Li, T., Zhang, C.: Oscillation of second-order differential equations with a sublinear neutral term. Carpath. J. Math. 30, 1-6 (2014)

7. Grace, S.R., Graef, J.R.: Oscillatory behavior of second order nonlinear differential equations with a sublinear neutral term. Math. Model. Anal. 23(2), 217-226 (2018)

8. Tamilvanan, S., Thandapani, E., Dzurina, J.: Oscillation of second-order neutral differential equation with sub-linear neutral term. Differ. Equ. Appl. 9(1), 29-35 (2017)

9. Agarwal, R.P., Bohner, M., Li, T., Zhang, C.: Hille and Nehari type criteria for third-order delay dynamic equations. J. Differ. Equ. Appl. 19(10), 1563-1579 (2013)

10. Erbe, L., Peterson, A., Saker, S.H.: Hille and Nehari type criteria for third-order dynamic equations. J. Math. Anal. Appl. 329(1), 112-131 (2007)

11. Agarwal, R.P., Bohner, M., Li, T., Zhang, C.: A philos-type theorem for third-order nonlinear retarded dynamic equations. Appl. Math. Comput. 249, 527-531 (2014)

12. Agarwal, R.P., Bohner, M., Tang, S., Li, T., Zhang, C.: Oscillation and asymptotic behavior of third-order nonlinear retarded dynamic equations. Appl. Math. Comput. 219(8), 3600-3609 (2012)

13. Han, Z., Li, T., Sun, S., Cao, F.: Oscillation criteria for third order nonlinear delay dynamic equations on time scales. Ann. Pol. Math. 99(2), 143-156 (2010)

14. Li, T., Han, Z., Sun, S., Zhao, Y.: Oscillation results for third order nonlinear delay dynamic equations on time scales. Bull. Malays. Math. Sci. Soc. 34(3), 639-648 (2011)

15. Yang, J.: Oscillation criteria for certain third-order delay dynamic equations. Adv. Differ. Equ. 2013, 178 (2013)

16. Grace, S.R.: Oscillatory behavior of third-order nonlinear difference equations with a nonlinear nonpositive neutral term. Mediterr. J. Math. 16, 128 (2019)

17. Candan, T:: Asymptotic properties of solutions of third-order nonlinear neutral dynamic equations. Adv. Differ. Equ. 2014, 35 (2014)

18. Chatzarakis, G.E., Grace, S.R., Jadlovska, I., Li, T., Tunc, E.: Oscillation criteria for third-order Emden-Fowler differential equations with unbounded neutral coefficients. Complexity 2019, Article ID 5691758 (2019)

19. Li, T., Rogovchenko, Y.V.: On the asymptotic behavior of solutions to a class of third-order nonlinear neutral differential equations. Appl. Math. Lett. 105, Article ID 106293 (2020)

20. Ganesan, V., Kumar, S.: Oscillation theorems for third-order retarded differential equations with a sublinear neutral term. Int. J. Pure Appl. Math. 114(5), 63-70 (2017)

21. Došlá, Z., Liška, P.: Oscillation of third-order nonlinear neutral differential equations. Appl. Math. Lett. 56, $42-48$ (2016)

22. Dzurina, J., Thandapani, E., Tamilvanan, S.: Oscillation of solutions to third-order half-linear neutral differential equations. Electron. J. Differ. Equ. 2012, 29 (2012)

23. Hassan, T.S., Agarwal, R.P., Mohammed, W.W.: Oscillation criteria for third-order functional half-linear dynamic equations. Adv. Differ. Equ. 2017, 111 (2017). https://doi.org/10.1186/s13662-017-1164-8

24. Karpuz, B.: Asymptotic behaviour of bounded solutions of a class of higher-order neutral dynamic equations. Appl. Math. Comput. 215(6), 2174-2183 (2009) 
25. Karpuz, B.: Sufficient conditions for the oscillation and asymptotic behaviour of higher-order dynamic equations of neutral type. Appl. Math. Comput. 221, 453-462 (2013)

26. Karpuz, B.: Comparison tests for the asymptotic behaviour of higher-order dynamic equations of neutral type. Forum Math. 27(5), 2759-2773 (2015)

27. Li, T., Han, Z:: Oscillation criteria for third-order nonlinear delay dynamic equations on time scales. Bull. Math. Anal. Appl. 3(1), 52-60 (2011)

28. Shi, Y., Han, Z., Hou, C.: Oscillation criteria for third-order neutral Emden-Fowler delay dynamic equations on time scales. J. Appl. Math. Comput. 55(1-2), 175-190 (2017)

29. Wang, Y., Han, Z., Sun, S., Zhao, P.: Hille and Nehari-type oscillation criteria for third-order Emden-Fowler neutral delay dynamic equations. Bull. Malays. Math. Sci. Soc. 40(3), 1187-1217 (2017)

30. Yao, J., Zhang, X., Yu, J.: New oscillation results for third-order half-linear neutral differential equations. arXiv:2001.01415v1 [math CA] (2020)

31. Zhang, S.Y., Wang, Q.R.: Oscillation of second-order nonlinear neutral dynamic equations on time scales. Appl. Math. Comput. 216, 2837-2848 (2010)

Submit your manuscript to a SpringerOpen ${ }^{\circ}$ journal and benefit from:

- Convenient online submission

- Rigorous peer review

- Open access: articles freely available online

- High visibility within the field

- Retaining the copyright to your article

Submit your next manuscript at $\boldsymbol{\nabla}$ springeropen.com 